„FÜR DIE MODERNEN METHODEN IN DEN LEBENSWISSENSCHAFTEN,

DIE IM WAHRSTEN SINNE DES WORTES DIE WELT BEWEGEN, BRAUCHEN

WIR EINE AGENDA, DIE VON EINER INTERNATIONALEN GEMEINSCHAFT

GEPRÜFT, GETRAGEN UND UMGESETZT WIRD.“

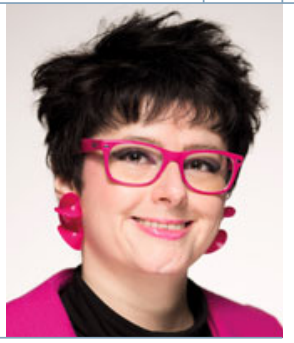

Dorothea Kaufmann

\title{
Freier Zugang zu Schlüsseltechnologien in den Life Sciences!
}

DOI: $10.1007 / \mathrm{s} 12268-018-0926-5$

(C) Springer-Verlag 2018

Wir können die Welt retten. Doch, wirklich! Alles, was wir dazu brauchen, halten wir mit modernen Schlüsseltechnologien wie CRISPR/ Cas 9 in Händen. Doch kommt es sehr darauf an, wem diese Hände gehören - noch gibt es keine weltweit einheitliche Gesetzgebung. Und während bei uns in Deutschland noch diskutiert wird, ob diese neue Form des Genetic Engineering mit dem (aktuell gültigen) Embryonenschutzgesetz vereinbar ist, werden in China und vermutlich vielen anderen Ländern schon die ersten Therapieversuche an Menschen unternommen. Doch wie sollen wir Wissenschaftlerinnen und Wissenschaftler nun mit diesem Dilemma umgehen? Darauf warten, dass sich alle Staaten einigen, wie mit modernen Technologien umzugehen ist; darauf hoffen, dass ein internationaler Konsens zum „Wohle der Menschheit“ gefunden wird oder doch resignieren, weil wir sowieso wissen, dass all dies nicht passieren wird?

Wem gehört eigentlich CRIPR/Cas9? Nach wie vor ist nicht abschließend geklärt, ob und - falls ja - wem welches Patent für die Entdeckung der Technik CRISPR/Cas9 erteilt wird. Da es sich hierbei um einen natürlich vorkommenden Mechanismus handelt, kann nicht dieser an sich patentiert werden, sondern „nur“ dessen verschiedene Anwendungsformen [1]. Die ganze Geschichte begann im Jahr 2004 mit dem US-Patent 7919277, in dem die Sequenzierung bestimmter CRISPRRegionen zur Unterscheidung verschiedener Varianten von Lactobacillus acidophilus benutzt wurde [2]. Zahlreiche Patente zu weiteren Anwendungen folgten; allerdings ohne große mediale Aufmerksamkeit.

Im Jahr 2012 nahm die Sache dann Schwung auf - kurz nacheinander reichten am 20. März Virginijus Šikšnys von der Universität Vilnius in Litauen [3], am 25. Mai Jennifer Doudna von der University of California zusammen mit Emmanuelle Charpentier vom Max-PlanckInstitut für Infektionsbiologie [4] sowie am 12. Dezember Feng Zhang vom Broad Institute of MIT and Harvard [5] Patentanträge zur Nutzung von CRISPR/Cas9 ein. Das U. S.
Patent and Trademark Office (PTO) erteilte schlussendlich das Patent auf die Anwendung in Eukaryoten Zhang, obwohl sein Antrag als Letzter eingereicht wurde. Hieraus entwickelte sich ein öffentlichkeitswirksamer Streit zwischen den beiden letztgenannten Parteien; Šikšnys zog es vor, seine Gedanken zu den Anwendungsmöglichkeiten von CRIPR/Cas9 DuPont zu überschreiben [6]. Mittlerweile hat sich DuPont auch mit dem Broad Institute [7] und Caribou Bioscience [8], der Firma von Doudna, verpartnert und hält so nun einen Großteil der Patente.

Im Alltag der Forscher*innen spielen diese aktuell (noch) keine Rolle, jede*r von uns kann CRIPR/Cas9 frei verwenden. Doch wird dies immer so bleiben? Auch wenn der Ansatz löblich ist, via Addgene CRISPR-Konstrukte für die Wissenschaft schnell und unkompliziert zu lizensieren, wird dieser auf lange Sicht vermutlich nicht gegen die Macht des Marktes bestehen können.

Dabei könnte alles so einfach sein. Nehmen wir an, z. B. die WHO würde alle Patente auf Schlüsseltechnologien wie CRIPSR/Cas9 innehaben und Wissenschaftler*innen weltweit hätten freien Zugang zu der Methode ihrer Wahl - vorbehaltlich der Prüfung durch eine Ethikkommission. Alleine der Umstand, dass eine mächtige, multinationale Vereinigung wie die WHO über Wohl und Wehe wacht, wäre ein Zeichen, das international verstanden werden würde.

Momentan ist die Wissenschaftswelt in kollektiver Schockstarre ob solcher Fragen, aber diese Frage wird sicher nicht die einzige bleiben, die David Collingridge heute bei seinen Ausführungen zur Technikfolgenabschätzung im Sinn haben könnte [9]. Genau für diese im wahrsten Sinne des Wortes weltbewegenden Fragen brauchen wir Antworten, die von einer internationalen Gemeinschaft geprüft, getragen und umgesetzt werden.

Ebenfalls wäre so die Übermacht und Monopolstellung von einzelnen Firmen unmöglich. Erinnern wir uns an die Rechtsstreitigkeiten um die Laboranwendung der Polymerasekettenreaktion (PCR) oder an den Einsatz der Grünen Gentechnik, die so lange ein Zukunftsver- sprechen war, bis Firmen wie Monsanto mit ihrer Geschäftspraxis das gesellschaftliche Ansehen der Methode so sehr in Verruf gebracht haben, dass dieser Forschungszweig zumindest in Deutschland nur noch ein Nischendasein fristet und gesellschaftlich stigmatisiert wird. So etwas darf nicht noch einmal passieren, weswegen wir uns alle gemeinsam dafür einsetzen müssen, die weitreichenden Folgen von Genetic Engineering im soziokulturellen und auch ethischen Bereich mit der Gesellschaft als solcher zu diskutieren, hinweg über alle Grenzen und mit der Hilfe der internationalen Staatengemeinschaft. Lassen Sie uns also für eine Wissenschaft einstehen, die dem Menschen dient, und nichts unversucht lassen, den Zugang zu Schlüsseltechnologien auch auf lange Sicht fair, nachhaltig, zugänglich und vor allem sicher zu gestalten.

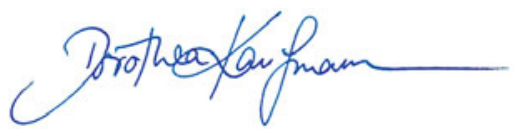

Dorothea Kaufmann,

Studienkoordinatorin Molekulare Biotechnologie, Universität Heidelberg

Korrespondenzadresse:

Dr. Dorothea Kaufmann

Institut für Pharmazie und Molekulare

Biotechnologie (IPMB)

Ruprecht-Karls-Universität Heidelberg

Im Neuenheimer Feld 364

D-69120 Heidelberg

Tel.: 06221-545670

D.Kaufmann@uni-heidelberg.de

\section{Quellen:}

[1] Dick A (2009) Erfindungen im Life Science-Bereich. BIOspektrum 2:207-208

[2] https://patents.google.com/patent/US7919277

[3] https://patents.google.com/patent/US9637739B2

[4] https://patents.google.com/patent/WO2013176772A1

[5] https://patents.google.com/patent/US8697359B1

[6] www.prweb.com/releases/dupont-pioneer-seed/vilnius-

university-cas 9/prweb12804075.htm

[7] www.broadinstitute.org/news/dupont-pioneer-and-broadinstitute-join-forces-enable-democratic-crispr-licensing-agriculture

[8] www.pioneer.com/home/site/about/news-media/newsreleases/template.CONTENT/guid.00DA8BCD-A60A-E6960179-E5BC63309087

[9] Collingridge D (1980) The Social Control of Technology. Pinter, London 